\title{
Research Paper: The Correlation Between Computerized Cognitive Tests Scores With Functional Cognitive Tests in People With Multiple Sclerosis
}

\author{
Elyas Monfared $^{1}$ (D), Hojjat Allah Haghgoo ${ }^{1^{*}}$ (D), Ebrahim Pishyareh ${ }^{1}$ (D), Mohsen Vahedi ${ }^{2}$ (D)
}

1. Department of Occupational Therapy, School of Rehabilitation, University of Social Welfare and Rehabilitation Sciences, Tehran, Iran

2. Department of Biostatistics and Epidemiology, School of Rehabilitation, University of Social Welfare and Rehabilitation Sciences, Tehran, Iran

\begin{tabular}{|c|c|}
\hline $\begin{array}{l}\text { Use your device to scan } \\
\text { and read the article online }\end{array}$ & Citation Monfared E, Haghgoo HA, Pishyareh E, Vahedi M. The Correlation Between Computerized Cognitive Tests \\
\hline 口ifistra & $\begin{array}{l}\text { Scores With Functional Cognitive Tests in People With Multiple Sclerosis. Iranian Rehabilitation Journal. 2022; 20(Special } \\
\text { Issue):79-88. http://dx.doi.org/10.32598/irj.20.SpecialIssue.1396.1 }\end{array}$ \\
\hline 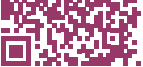 & doi'http://dx.doi.org/10.32598/irj.20.SpecialIssue.1396.1 \\
\hline
\end{tabular}

Article info:

Received: 09 Apr 2021

Accepted: 17 May 2021

Available Online: $01 \mathrm{Jam} 2022$

\section{Keywords:}

Occupational Therapy,

Multiple Sclerosis, Cognition,

Neuropsychological Tests,

Visual Perception

\section{ABSTRACT}

Objectives: In order to rehabilitate cognitive disorders, it is necessary to carefully evaluate cognitive function. Given the variety of assessments, including computerized and functional tests, the aim of this study was to determine whether computerized test scores really reflect a person's functional ability. In this study, we measured the correlation between computerized and functional evaluation results

Methods: In this cross-section study, 45 people ( 9 males and 36 females) with multiple sclerosis with an Expanded Disability Score (EDSS) of 1 to 5 were recruited. To assess their cognitive function, the CANTAB tests (SOC, DMS, and SSP tests) and the Loewenstein Occupational Therapy Cognitive Assessment (LOTCA) battery were used. The data were then processed with SPSS software v. 22.

Results: The results showed that the CANTAB test is well correlated with the LOTCA battery. A high correlation was observed between LOTCA's "visual perception" with delayed matching to sample ( $r=0.909)$, LOTCA's "spatial perception" with spatial span test $(r=0.907)$, LOTCA's "visual organization" with stocking of Cambridge $(\mathrm{r}=0.961)$, and "mental operations" in LOTCA with "stocking of Cambridge" ( $\mathrm{r}=0.835)$.

Discussion: Due to the very good convergence of these two tests, in many cases, computerized tests can be used instead of functional tests.

\footnotetext{
* Corresponding Author:
}

Hojjat Allah Haghgoo, PhD.

Address: Department of Occupational Therapy, School of Rehabilitation, University of Social Welfare and Rehabilitation Sciences, Tehran, Iran Tel: +98 (990) 1245852

E-mail:h.haghgoo@yahoo.com 


\section{Highlights}

- Computerized test scores really reflect a person's functional ability.

- The CANTAB test is well correlated with the LOTCA battery.

- The LOTCA's "visual perception" with delayed matching to sample in CANTAB

- The LOTCA's "spatial perception” with spatial span test in CANTAB

- The LOTCA's“visual organization” with stocking of Cambridge in CANTAB

- The "mental operations" in LOTCA with "stocking of Cambridge" in CANTAB

- The Computerized tests can be used instead of functional tests.

\section{Plain Language Summary}

People with central nervous system disorders develop cognitive impairments. To help these people, it is necessary to carefully evaluate their cognitive functions. The tools for assessing these disorders are usually performance-based and their scoring is subjective and general. Excessive fatigue in diseases such as multiple sclerosis can severely affect the results of functional evaluation. Computer evaluations, on the other hand, are both less tedious and more accurate to score. The purpose of this study was to determine whether computer test scores accurately indicate a person's function. In this study, the functional scores of 45 patients with multiple sclerosis were compared with their computer scores. An occupational therapy test (LOTCA) was used to evaluate them, and computerized tests developed by the University of Cambridge (named CANTAB) were used for computer evaluation. The results showed that there is a high correlation between people's scores in the two types of assessments. Due to the strong convergence of these two tests, in many cases, as an alternative, computerized tests can replace functional tests. That is, depending on the patient's condition and ability to use any of these tests, similar results are obtained. In situations where it is difficult to use functional assessment due to the patient's physical problems, computerized assessments easily solves this problem. These tools are great alternatives to performance assessment tools.

\section{Introduction}

ognition involves all the mental pro-

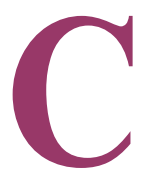
cesses that underlie recognition, learning, remembering, and considering variable information [1]. Cognition refers to design, problem-solving, awareness, and judgment, which are classified as higher cognitive functions. Each cognitive system is a series of processing steps that work together to achieve a common goal. Cognitive systems involve in functional activities. In people with central nervous system disorders, cognitive abilities are impaired and their performance is affected [2]. Cognitive impairment is a temporary or permanent defect in the processing, storage, and retrieval of information required by a person to perform normal activities in a safe manner or in proportion to his or her age and educational and cultural context. These defects can lead to restrictions in all activities of a person's daily life.
These include activities of daily life, education, work, play, leisure, and social participation [3].

Multiple Sclerosis (MS), as a neurological disorder, is a progressive disease of the central nervous system characterized by diffuse lesions in the brain and spinal cord that cause physical disabilities accompanying cognitive impairments. MS has various effects on different aspects of cognitive function, including attention, information processing efficiency, executive functions, processing speed, long-term memory, and visual perception [4]. Cognitive impairment also affects the patient's behavior, social participation, adaptive strategies, and causes functional limitations in the people's performance that greatly affect their activities of daily living performances $[5,6]$.

A large cross-sectional study conducted in nine European countries reported that only $35.8 \%$ of People with MS (PwMS) are employed. Low mood and cognitive prob- 
lems affect memory, attention, and information processing speed, which have been repeatedly reported for job-related problems. Among these, memory impairment is the most common cause of unemployment among PwMS [7]. Recent research showed that $20 \%$ of PwMS have social cognitive impairments and social cognitive impairment $[8,9]$ is significantly associated with the performance of other cognitive domains, such as working memory, information processing speed, and executable functions [10].

Cognitive impairment not only affects patients but also affects their relationships with their families and causes repeated complaints from caregivers [6]. Therefore, accurate cognitive assessment is necessary for cognitive intervention, which should be prioritized along with other therapies [11]. Both the evaluation and intervention processes are in a dynamic interaction that enables people to engage in the desired activities in the natural context. Cognitive function assessment in PwMS should be part of clinical practice as a result of clinical trials. Ideally, any patient diagnosed with MS should undergo a complete and precise neuropsychiatric evaluation and be re-evaluated regularly using standard and valid sets so that significant clinical changes can be documented as well as early intervention implemented. Therefore, it is necessary to select appropriate tools and methods for evaluation as an important process of the treatment [12]. Various methods are used for evaluation, including computerized and functional evaluations [13].

Functional assessments result in more objective and qualitative outcomes [1]. Computerized tests, on the other hand, provide accurate scores [14]. Some evaluations, data collection, interpretation, and documentation of reports may be ineffective as long as they rely on human observation and individual interpretation of the results. All of these operations can be performed more efficiently using a computer [15]. Most studies comparing computerized and functional tests focus on scores and computer scores are more accurate than functional scores. Subjects may also take computerized tests faster [16]. On the other hand, some have suggested that people with fewer keyboard skills perform worse on computer-based tests [17]. In some cases, subjects spend more time on computer tests than on functional tests [18]. Also, the physical nature of the computer screen can lead to reduced performance compared to performance tests, which can be attributed to additional information processing [19]. Day by day, many tests turn into computer tests, because accurate measurements are always more desirable and documented than subjective measurements [20].
Given the importance of assessment in occupational therapy and the nature of some tests that require a special environment and conditions that may not be applicable in all cases, there is a need to use simpler available and alternative assessments and the value, power, and accuracy of different cognitive assessments tools should be assessed. This enables us to consider the results of the evaluation with high reliability.

In this study, we investigated the question of whether computer-based test results are correlated with the results of functional tests in patients' cognitive abilities. Therefore, the correlation between cognitive performance evaluation results in PwMS was examined in both computer-based and functional methods.

\section{Materials and Methods}

All participants were provided with the information sheet and ensured that their participation in the research is voluntary and they were able to withdraw from the study at any stage of the data collection process. Following their consent, data were collected at the participant's convenient time and day. All PwMS who provided consent forms were included in the study.

\section{Participants}

In this cross-sectional study, 45 PwMS (36 women and 9 men) under the age of 70 years from two clinical settings specialized for these people, i.e., Hazrat Abolfazl Rehabilitation Clinic and MS Center in Isfahan were recruited. They were selected based on the following inclusion criteria: 1- willingness to participate in research, 2-definitive diagnosis of MS by a neurologist, 3- EDSS score below 5 (diagnosed by a neurologist), 4-absence of intellectual disorders and fractures, 5-ability to work with a computer, 6- no use of corticosteroids medication 28 days before the test $[21,22], 7$ - no history of other neurological diseases, 8- no addiction, and 9- the ability to give informed consent.

The subjects were excluded if they did not complete the tests, or in the case of recurrence of the attack while completing the tests. In a consecutive manner, the participants were randomly assigned to two groups 1 and 2 . Group 1 was assessed by the Lowenstein Occupational Therapy Cognitive Assessment (LOTCA) at the first session and Cambridge Neuropsychological Test Automated Battery (CANTAB) in the second session, while the second group, was first assessed by CANTAB and then by LOTCA. 


\section{Data collection}

Data collection was conducted between 9 and 12 PM to avoid fatigue effects on the results. There were three tools for data collection, including:

A questionnaire to collect data on age, gender, educational level, employment status, marital status, family history, type of MS, the participant's score in EDSS, and history of other psychological/physical disorders.

The Lowenstein Occupational Therapy Cognitive Assessment (LOTCA) test was used to assess functional cognitive abilities. LOTCA is a set of tests designed in 1990 for occupational therapies to assess a person's cognitive processes and determine how well a person is able to perform his daily functional tasks [1]. It is applicable for patients with brain injury, stroke survivors, spinal cord injury, people with dementia, patients with brain tumors, patients with central nervous system injuries, such as MS, people with mental illness, and children with learning disabilities. LOTCA test can be used for people aged 6 to 70 years old.

The original LOTCA test included 20 subtests in four areas: awareness, participation, visuomotor organization, and thinking operations. However, LOTCA's latest edition includes 26 sub-tests in six parts, including orientation, visual perception, space perception, motion proxy, visuomotor organization, and thinking operations. The orientation subtest consists of two items: temporal and spatial orientation, each of which is scored between 1 and 8 . A higher score on this test indicates better performance of the orientation. LOTCA's visual perception test includes four items: a person's ability to identify images of everyday objects, images of objects from unusual angles, overlapping images, and spatial relationships between objects, all of which are rated between 1 and 4 . A higher score indicates a person's better performance in visual perception.

The LOTCA's space perception subtest the LOTCA's space perception subscale includes three items to assess the ability to distinguish between right and left, and to determine the spatial relationship between objects and oneself.. All three test items in this section are scored between 1 and 4 , and a higher score in this section indicates a person's better performance in spatial perception. LOTCA's motor proxy test consists of three items: a person's ability to imitate a movement, use objects, and perform symbolic actions.

All three tests in this section are graded between 1 and 4 , and a higher score indicates a person's better perfor- mance in the motor proxy. LOTCA's visual organizational subtest includes seven items: one's ability to copy geometric shapes, produce a two-dimensional model, copy a color block design and design a simple block, build a puzzle, complete a pegboard, and draw a clock. All parts of the test are scored between 1 and 4, with a higher score indicating better performance in the visuomotor organization.

The thinking operation subtest also includes seven items: classification test, logical questions, object classification, structural subtest, first pictorial ordering, second pictorial ordering (if the patient scores 4 in the first pictorial ordering), geometric ordering, and the attention and concentration. Except for the first three items that are graded between 1 and 5, the rest of them are scored between 1 and 4, and the higher a person's score in this section, the better the person's performance in thinking operations [23]. The validity and reliability of this test were assessed in 1989 [1]. Construct validity of the Persian version was assessed in 2009 [24]. In this study, we used the Persian version of LOTCA and all items of this test were used to collect information.

The CANTAB test is a set of computerized neuropsychological tests developed at the University of Cambridge that is used to assess the components of cognition, especially those associated with the anterior and middle temporal lobe and frontal lobe functions. This set consists of 22 neuropsychological tests in the cognitive domains, such as motor skills, visual attention, Visual/spatial memory, and working memory, decision-making, response control, executive function, and attention. The subject responds to the tests by touching the computer screen, and the results are scored by the computer. All tests were non-verbal and their validity has been supported [25].

The validity and reliability of this test were assessed in 2006 for Central Nervous System (CNS) lesions, such as MS [26]. In this study, we used three tests from the set of CANTAB tests, including SSP (Spatial Span Test), SOC (Stocking of Cambridge), and DMS (Delayed Matching to Sample) tests. The SSP test evaluates working memory and short-term memory, which is a frontal lobe performance evaluation. In the SSP test, there are six criteria that can be grouped into four categories: the number of correct answers, the number of errors, the number of attempts, and the delay [27].

In this study, the score obtained from the number of correct answers was selected as the variable criterion. The higher the number of correct answers, the higher the score, which indicates better performance [1]. The 
SOC test is about spatial planning, which encourages people to use problem-solving strategies as well as visual matching and perception of two sets of stimuli. The test also evaluates working memory and assesses the function of the frontal lobe. In the SOC test, there are four variables, including the ability to solve problems in the least movement, the average of $2,3,4$, and 5 movements to solve the problem, the delay in movement, and the amount of thinking time to solve problems [27, 28]. In this study, the score of problem-solving ability in the least movement was selected as the criterion for change. The higher the number of correct answers in the smallest movement, the higher the score on the test, which indicates better problem-solving ability and better performance [1]. The DMS test is primarily sensitive to damage to the middle temporal lobe area and then to entering projections from the frontal lobe. This test assesses the ability to simultaneously match the vision and short-term memory for nonverbal patterns. In the DMS test, there are 14 variables that can be divided into three groups: response delay, the number and percentage of correct and incorrect answers, and theoretical measures to identify the response [25]. In this study, the number and percentage of correct answers were selected as the criterion for change. The higher the number of correct answers in the test, the higher the score that indicates better performance [1].

\section{Statistical analysis}

In this study, descriptive statistics, such as mean, standard deviation, and percentages were used to describe the data. The Kolmogorov-Smirnov test was used to test the normality of the data, the Pearson correlation test was used to examine the correlation between variables with normal distribution, and the Spearman correlation coefficient for non-parametric data. The significance level was considered to be 0.05 . SPSS software version 23 was used to analyze the data.

\section{Results}

A total of 63 people diagnosed with MS participated in this study. After evaluating them according to the criteria, 48 cases met the criteria and entered the study. Of these, three individuals did not complete all the assessments and were excluded. Forty-five people (36 women and 9 men) with an average age of 40 years (ranging from 19-69 years old) completed all assessments. While the education level of participants ranged from below the diploma to above the bachelor's degree, most of them (more than 73\%) had a bachelor's or master's degree. About $38 \%$ of the subjects were employed, of whom
$13 \%$ were students. This may indicate that there are not many jobs for patients with the disease. Only 12 participants reported a history of the disease in their family. The type of MS in most of the participants was a relapse and remitting (RRMS). Less than $25 \%$ of their MS type was the primary and secondary progressive and progressive-relapsing types.

Table 1 shows the results of the Spearman correlation coefficient between the LOTCA subscales and CANTAB tests. According to Table 1, all three tests of the CANTAB well correlated with all six parts of the LOTCA test. The results included a very high correlation between "visual perception" and "delayed matching to sample' (r=0.909), "spatial perception" and "spatial span test" $(\mathrm{r}=0.907)$, "visuomotor organization" and "Stocking of Cambridge" ( $\mathrm{r}=0.961)$, and "thinking operations" and stocking of Cambridge $(\mathrm{r}=0.835)$. It is worth noting that the "thinking operation" was highly correlated with all three CANTAB measures.

Multiple linear regression was calculated to model the relationship between the LOTCA subscales and the CANTAB test scores (SOC, SSP, and DMS). Table 2 shows the intensity and relevance of the SOC scores to the LOTCA subscales.

Table 2 shows that the SOC scores had a significant positive correlation with all LOTCA subscales. However, it was more correlated with "visuomotor organization" and "thinking operations". For example, for a unit increase in SOC score, LOTCA's "thinking operation" score increases by 1.539 points. The SOC scores had low but significant power to predict performance in orientation, visual perception, spatial perception, and motor praxis while showing high and significant power to predict visuomotor organization and thinking operation in the LOTCA test.

Table 3 indicates that the SSP scores had a significant positive correlation with all LOTCA subscales. But its better correlations are with "Spatial perception", "Visumotor Organization" and "thinking operations", respectively. For example, for a unit increase in SOC score, LOTCA's "thinking operation" score increases by 1.7 points. The SSP scores had low but significant power to predict performance in orientation, visual perception, and motor praxis while showing high and significant power to predict spatial perception, visuomotor organization, and thinking operation in the LOTCA test.

Table 4 indicates that the SSP scores have significant positive correlations with all LOTCA subscales. How- 
Table 1. Correlation between LOTCA subscales and CANTAB tests

\begin{tabular}{lccc}
\hline LOTCA Subscales & SOC & SSP & DMS \\
\hline Orientation & $r=0.373, P=0.012$ & $r=0.395, P=0.007$ & $r=0.672, P<0.001$ \\
\hline Visual perception & $r=0.365, P=0.014$ & $r=0.483, P=0.001$ & $r=0.909, P<0.001$ \\
\hline Spatial perception & $r=0.477, P=0.001$ & $r=0.907, P<0.001$ & $r=0.378, P=0.010$ \\
\hline Motor praxis & $r=0.509, P<0.001$ & $r=0.572, P<0.001$ & $r=0.830, P<0.001$ \\
\hline Visumotor organization & $r=0.961, P<0.001$ & $r=0.598, P<0.001$ & $r=0.358, P=0.016$ \\
\hline Thinking operation & $r=0.835, P<0.001$ & $r=0.701, P<0.001$ & $r=0.654, P<0.001$ \\
\hline
\end{tabular}

Iranian Rehabilitation Dournal

LOTCA: Loewenstein Occupational Therapy Cognitive Assessment; SOC: Stocking of Cambridge ; SSP: Spatial Span Test ; DMS: Delayed Matching to Sample

ever, it was more correlated with "thinking operations". For example, for a unit increase in the DMS score, the "Thinking Operation" score increases by 0.165 points. The DMS scores had low but significant power to predict performance in orientation, visual perception, spatial perception, motor praxis, and visuomotor organization while showing higher and significant power to predict the "thinking operation" in the LOTCA test.

\section{Discussion}

In this study, the relations between six LOTCA subtests were calculated by SOC, SSP, and DMS tests. In this section, the results of the performance evaluated in the orientation subtest were examined with control tests.

The "visual perception" subtest showed the most correlation with the DMS test in CANTAB. The DMS test evaluates visual adaptation and short-term memory. It is sensitive to the inputs from the medial temporal lobe and frontal lobe [29]. Therefore, according to the results, it can be concluded that the DMS test has good potentiation to evaluate the factors of visual perception items in LOTCA. The LOTCA's "vision perception" subtest also is highly correlated with SSP and SOC, respectively.

The "orientation" subtest of the LOTCA showed the greatest correlation with the DMS test. Therefore, the DMS test has good potential to evaluate the "orientation" in the LOTCA test, which includes the temporal and spatial orientation. LOTCA's "orientation" subtest also showed relatively good correlations with the other two CANTAB tests.

The "spatial perception" test showed the most correlation with the SSP test in CANTAB. The SSP test evalu-

Table 2. Prediction power of the SOC scores to the LOTCA subtests

\begin{tabular}{|ccccc|}
\hline LOTCA Subscales/SOC & Co-Efficient & $\begin{array}{c}\text { 95\% Confidence } \\
\text { Interval }\end{array}$ & Standard Error & P \\
\hline Orientation & 0.098 & $0.030-0.166$ & 0.034 & 0.006 \\
\hline Visual perception & 0.209 & $0.064-0.354$ & 0.072 & 0.006 \\
\hline Spatial perception & 0.315 & $0.162-0.468$ & 0.076 & $<0.001$ \\
\hline Motor praxis & 0.332 & $0.162-0.503$ & 0.085 & $<0.001$ \\
\hline Visumotor organization & 1.436 & $1.306-1.556$ & 0.065 & $<0.001$ \\
\hline Thinking operation & 1.539 & $1.278-1.799$ & 0.129 & $<0.001$ \\
\hline
\end{tabular}


Table 3. Prediction power of the SSP scores to the LOTCA subtests

\begin{tabular}{|ccccc|}
\hline LOTCA Subscales/SSP & Co-Efficient & $\begin{array}{c}\text { 95\% Confidence } \\
\text { Interval }\end{array}$ & Standard Error & P \\
\hline Orientation & 0.140 & $0241-0.308$ & 0.050 & 0.008 \\
\hline Visual perception & 0.352 & $0.144-0.560$ & 0.103 & 0.001 \\
\hline Spatial perception & 0.722 & $0.573-0.872$ & 0.074 & $<0.001$ \\
\hline Motor praxis & 0.506 & $0.257-0.754$ & 0.123 & $<0.001$ \\
\hline Visumotor organization & 1.279 & $0.747-1.874$ & 0.273 & $<0.001$ \\
\hline Thinking operation & 1.702 & $1.100-2.303$ & 0.298 & $<0.001$ \\
\hline
\end{tabular}

Iranian Rehabilitation Journa

ates working memory and short-term memory, as an assessment of the frontal lobe function [27]. Therefore, it can be concluded that the SSP test has a high potential for evaluating the factors considered in the "spatial perception" subtest of the LOTCA. Also, the "spatial perception" test has high correlations with SOC and DMS after SSP, respectively.

The "motor praxis" test showed more correlation with the DMS test. Thus, the DMS test has a high power to evaluate the motor praxis subtest of LOTCA. Also, the LOTCA motor praxis test showed high correlations with SSP and SOC, respectively. The results showed that using each of the three subtests (SOC, SSP, and especially DMS tests) can predict one's function in motor praxis.

The "visuomotor organizational" subtest showed the greatest correlation with the SOC test. The SOC test assesses spatial planning, which encourages people to use problem-solving strategies as well as visual matching and perception of two sets of stimuli. The test also evaluates working memory and the function of the frontal lobe [26]. Therefore, the SOC test has a high power to evaluate the "visuomotor organization" item. Also, the visuomotor organization test showed high correlations with SSP and DMS, respectively.

The "thinking operation" subtest showed the highest correlation with the SOC test. Therefore, the SOC test has also good conditions for evaluating the factors of the "thinking operation". Also, the "thinking operation" test showed high correlations with the SSP and DMS tests, respectively. Analysis of the results of the LOTCA and CANTAB tests in the evaluated MS subjects showed that all six subtests of the LOTCA test are in good convergence with the CANTAB tests.

According to these findings, there were good correlations between the functional test of LOTCA and the computerized test of CANTAB in PwMS. These findings suggest that computer evaluation tools can also well predict performance capabilities. In 2007, Scherer reviewed several functional tests to measure cognitive function in patients with MS, stating that since time and cost savings are always important, therapists must select the best cognitive test to address each patient's problem [30]. Given this issue and the results of the present study, which showed high correlations and predictive power

Table 4. Prediction power of the DMS scores to the LOTCA subtests

\begin{tabular}{cccc}
\hline LOTCA Subscales/SSP & Co-Efficient & $\begin{array}{c}95 \% \text { Confidence } \\
\text { Interval }\end{array}$ & Standard Error \\
\hline $\begin{array}{c}\text { Orientation } \\
\text { Visual perception }\end{array}$ & 0.028 & $0.020-0.036$ & 0.004 \\
\hline Spatial perception & 0.072 & $0.061-0.083$ & 0.005 \\
\hline Motor praxis & 0.041 & $0.016-0.066$ & 0.012 \\
\hline Visumotor organization & 0.085 & $0.069-0.0101$ & 0.008 \\
\hline Thinking operation & 0.095 & $0.030-0.160$ & 0.032 \\
\hline
\end{tabular}

Mranian Rehabilitation Journa 
between the two computerized and functional tests, it can conclude that the use of both types of tests can be a priority in certain circumstances.

As mentioned earlier, computer tests provide more accurate quantitative results [14], they are cheaper and less time-consuming, but may be more stressful [31]. However, the level of participation in participation in computer tests is higher $[14,16]$. Some researchers suggest that because of the physical nature of the screen, which requires more information processing [19] or fewer keyboard skills [17], people's participation in functional tests is higher than in computer tests. The most important point is how to score on computer and performance tests. Functional tests are scored by humans and it is affected by human factors and many other mental conditions of the examiner, which can affect an individual's reported score, whereas, in computerized tests, human is not involved in scoring, and the subject gets an accurate score [32]. As Noyes stated in 2008 [33], the results of the correlation between computer and functional tests show contradictory results. Some studies have emphasized the existence of high convergence, and some have indicated a lack of convergence. This issue needs further investigation.

\section{Conclusion}

According to the results of the present study, in order to evaluate cognitive disorders and in cases where functional tests are not available or a person is unable to use them due to functional limitations, computer tests can be used instead of functional tests. Finally, given the size of the sample and other limitations, this study may be considered as a primary study, and to examine these results more precisely, studies with larger sample size and more facilities are suggested.

\section{Ethical Considerations}

\section{Compliance with ethical guidelines}

This study was approved by the ethical committee of the University of Social Welfare and Rehabilitation Sciences (Code: IR.USWR.REC.1399.016).

\section{Funding}

This research was supported by the University of Social Welfare and Rehabilitation.

\section{Authors' contributions}

All authors equally contributed in preparing this article.

\section{Conflict of interest}

The authors declared no conflict of interests.

\section{Acknowledgments}

The cooperation of all participants and their parents in this study is greatly appreciated.

\section{References}

[1] Katz N, Itzkovich M, Averbuch S, Elazar B. Loewenstein Occupational Therapy Cognitive Assessment (LOTCA) battery for brain-injured patients: Reliability and validity. American Journal of Occupational Therapy. 1989; 43(3):184-92 [DOI:10.5014/ajot.43.3.184] [PMID]

[2] Correa DD, Maron L, Harder H, Klein M, Armstrong CL, Calabrese $\mathrm{P}$, et al. Cognitive functions in primary central nervous system lymphoma: Literature review and assessment guidelines. Annals of Oncology. 2007; 18(7):1145-51. [DOI:10.1093/annonc/mdl464] [PMID]

[3] The American Journal of Occupational Therapy. Management of occupational therapy services for persons with cognitive impairments (statement). American Journal of Occupational Therapy. 1999; 53(6):601-7. [DOI:10.5014/ajot.53.6.601] [PMID]

[4] Langdon DW. Cognition in multiple sclerosis. Current Opinion in Neurology. 2011; 24(3):244-9. [DOI:10.1097/ WCO.0b013e328346a43b] [PMID]

[5] Deloire MS, Ruet A, Hamel D, Bonnet M, Dousset V, Brochet $B$. MRI predictors of cognitive outcome in early multiple sclerosis. Neurology. 2011; 76(13):1161-7. [DOI:10.1212/ WNL.0b013e318212a8be] [PMID] [PMCID]

[6] Schiavolin S, Leonardi M, Giovannetti AM, Antozzi C, Brambilla L, Confalonieri $\mathrm{P}$, et al. Factors related to difficulties with employment in patients with multiple sclerosis: A review of 2002-2011 literature. International Journal of Rehabilitation Research. 2013; 36(2):105-11. [DOI:10.1097/ MRR.0b013e32835c79ea] [PMID]

[7] Raggi A, Covelli V, Schiavolin S, Scaratti C, Leonardi M, Willems M. Work-related problems in multiple sclerosis: A literature review on its associates and determinants. Disability and Rehabilitation. 2016; 38(10):936-44. [PMID]

[8] Yamout B, Issa Z, Herlopian A, El Bejjani M, Khalifa A, Ghadieh AS, et al. Predictors of quality of life among multiple sclerosis patients: A comprehensive analysis. European Journal of Neurology. 2013; 20(5):756-64. [DOI:10.1111/ene.12046] [PMID]

[9] Dulau C, Deloire M, Diaz H, Saubusse A, Charre-Morin J, Prouteau A, et al. Social cognition according to cognitive impairment in different clinical phenotypes of multiple sclerosis. Journal of Neurology. 2017; 264(4):740-8. [DOI:10.1007/ s00415-017-8417-z] [PMID]

[10] Henry A, Tourbah A, Chaunu MP, Bakchine S, Montreui M. Social cognition abilities in patients with different multiple sclerosis subtypes. Journal of the International Neuropsychological Society. 2017; 23(8):653-64. [DOI:10.1017/ S1355617717000510] [PMID] 
[11] Stys PK. Myelocortical multiple sclerosis: A new disease subtype? The Lancet Neurology. 2018; 17(10):832-4 [DOI:10.1016/S1474-4422(18)30333-8]

[12] Macías Islas MÁ, Ciampi E. Assessment and impact of cognitive impairment in multiple sclerosis: An overview. Biomedicines. 2019; 7(1):22. [DOI:10.3390/biomedicines7010022] [PMID] [PMCID]

[13] Ottenbacher KJ, Cusick A. Discriminative versus evaluative assessment: Some observations on goal attainment scaling. American Journal of Occupational Therapy. 1993; 47(4):349-54. [DOI:10.5014/ajot.47.4.349] [PMID]

[14] Olsen JB, Maynes DD, Slawson D, Ho K. Comparisons of paper-administered, computer-administered and computerized adaptive achievement tests. Journal of Educational Computing Research. 1989; 5(3):311-26. [DOI:10.2190/86RK76WN-VAJ0-PFA3]

[15] Angelo J, Smith RO. An analysis of computer-related articles in occupational therapy periodicals. The American Journal of Occupational Therapy. 1993; 47(1):25-9. [DOI:10.5014/ ajot.47.1.25] [PMID]

[16] Greaud VA, Green BF. Equivalence of conventional and computer presentation of speed tests. Applied Psychological Measurement. 1986; 10(1):23-34. [DOI:10.1177/014662168601000102 ]

[17] Russell M. Testing on Computers. Education Policy Analysis Archives. 1999; 7:20. [DOI:10.14507/epaa.v7n20.1999]

[18] Bugbee Jr AC, Bernt FM. Testing by computer: Findings in six years of use 1982-1988. Journal of Research on Computing in Education. 1990; 23(1):87-100. [DOI:10.1080/08886504.1990 .10781945]

[19] Ziefle M. Effects of display resolution on visual performance. Human Factors. 1998; 40(4):554-68. [DOI:10.1518/001 872098779649355] [PMID]

[20] Bodmann SM, Robinson DH. Speed and performance differences among computer-based and paper-pencil tests. Journal of Educational Computing Research. 2004; 31(1):51-60. [DOI:10.2190/GRQQ-YTOF-7LKB-F033]

[21] Demaree HA, DeLuca J, Gaudino EA, Diamond BJ. Speed of information processing as a key deficit in multiple sclerosis: Implications for rehabilitation. Journal of Neurology, Neurosurgery \& Psychiatry. 1999; 67(5):661-3. [DOI:10.1136/ jnnp.67.5.661] [PMID] [PMCID]

[22] Hancock LM, Bruce JM, Bruce AS, Lynch SG. Processing speed and working memory training in multiple sclerosis: A doubleblind randomized controlled pilot study. Journal of Clinical and Experimental Neuropsychology. 2015; 37(2):113-27. [PMID]

[23] Itzkovich M, Elazar B, Averbuch S, Katz N. Loewenstein occupational therapy cognitive assessment (LOTCA) manual. Maddak Incorporated. 2000. https:/ / research.aota.org/ajot/ article-abstract/43/3/184/2259/Loewenstein-OccupationalTherapy-Cognitive

[24] Karbalaei-Nouri A, Sadeghi A, Shamsolma'aali Z. [Construct validity confirmation of Iranian version of Lowenstein Occupational Therapy Cognitive Assessment (LOTCA) (Persian)]. Archives of Rehabilitation. 2009; 10(2):1-5. http:// rehabilitationj.uswr.ac.ir/article-1-329-en.html
[25] Luciana M, Nelson CA. Assessment of neuropsychological function through use of the Cambridge Neuropsychological Testing Automated Battery: Performance in 4-to 12-year-old children. Developmental Neuropsychology. 2002; 22(3):595-624. [PMID]

[26] Gualtieri CT, Johnson LG. Reliability and validity of a computerized neurocognitive test battery, CNS Vital Signs. Archives of Clinical Neuropsychology. 2006; 21(7):623-43. [DOI:10.1016/j.acn.2006.05.007] [PMID]

[27] Torgersen J, Flaatten H, Engelsen BA, Gramstad A. Clinical validation of Cambridge neuropsychological test automated battery in a Norwegian epilepsy population. Journal of Behavioral and Brain Science. 2012; 2(1):108-16. [DOI:10.4236/ jbbs.2012.21013]

[28] Talebi M, Majdi A, Kamari F, Sadigh-Eteghad S. The Cambridge Neuropsychological Test Automated Battery (CAN$\mathrm{TAB}$ ) versus the Minimal Assessment of Cognitive Function in Multiple Sclerosis (MACFIMS) for the assessment of cognitive function in patients with multiple sclerosis. Multiple Sclerosis and Related Disorders. 2020; 43:102172. [DOI:10.1016/j. msard.2020.102172] [PMID]

[29] Giedraitiene N, Kaubrys G. Distinctive pattern of cognitive disorders during multiple sclerosis relapse and recovery based on computerized CANTAB tests. Frontiers in Neurology. 2019; 10:572. [DOI:10.3389/fneur.2019.00572] [PMID] [PMCID]

[30] Scherer P. Cognitive screening in multiple sclerosis. Journal of Neurology. 2007; 254(Suppl 2):II26-9. [DOI:10.1007/ s00415-007-2008-3] [PMID]

[31] Wästlund E, Reinikka H, Norlander T, Archer T. Effects of VDT and paper presentation on consumption and production of information: Psychological and physiological factors. Computers in Human Behavior. 2005; 21(2):377-94. [DOI:10.1016/j. chb.2004.02.007]

[32] Gyure WJ, Hoover AM. Completing functional testing. Google Patents; 2014. https://scienceon.kisti.re.kr/srch/selectPORSrchPatent.do?cn=USP2016119507697

[33] Noyes JM, Garland KJ. Computer-vs. paper-based tasks: Are they equivalent? Ergonomics. 2008; 51(9):1352-75. [PMID] 
This Page Intentionally Left Blank 